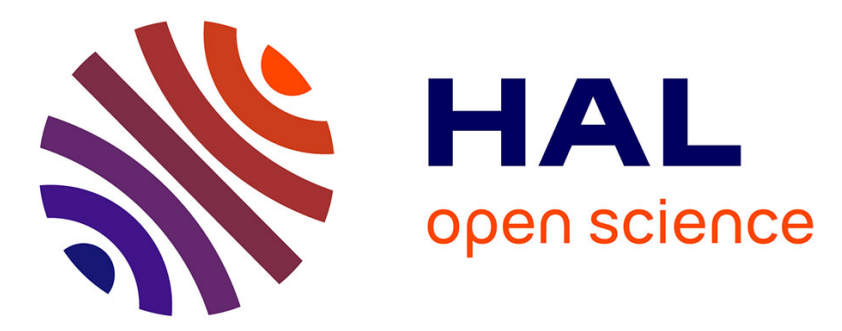

\title{
Pluralisme philosophique versus Logique intuitionniste Joseph Vidal-Rosset
}

\section{To cite this version:}

Joseph Vidal-Rosset. Pluralisme philosophique versus Logique intuitionniste. Al-Mukhatabat. Trilingual Journal for Logic, Epistemology and Analytic Philosophy, 2014, 11, pp.174-194. hal-01240911

\section{HAL Id: hal-01240911 \\ https://hal.science/hal-01240911}

Submitted on 9 Dec 2015

HAL is a multi-disciplinary open access archive for the deposit and dissemination of scientific research documents, whether they are published or not. The documents may come from teaching and research institutions in France or abroad, or from public or private research centers.
L'archive ouverte pluridisciplinaire HAL, est destinée au dépôt et à la diffusion de documents scientifiques de niveau recherche, publiés ou non, émanant des établissements d'enseignement et de recherche français ou étrangers, des laboratoires publics ou privés. 


\title{
Pluralisme philosophique versus Logique intuitionniste
}

\author{
Joseph Vidal-Rosset \\ (Université de Lorraine, Archives Poincaré, Nancy) \\ Pour Jacques Bouveresse
}

\begin{abstract}
In a first time, this paper shows why the classification of philosophical systems defined by Vuillemin improves our understanding of the history of philosophy. I reply in a second time to embarrassments expressed by Bouveresse and Engel about the philosophical pluralism which is, according to Vuillemin, a consequence of the principles and definitions of his classification of philosophical systems. The main thesis of this paper is a conjecture: the philosophical pluralism is not a position that one is forced to admit if one adopts Vuillemin's classification. Elements that should allow a proof of this conjecture are given in conclusion.
\end{abstract}

Keywords : Philosophy of logic, classical logic, intuitionnistic logic.

$$
\begin{aligned}
& \text { ملخّص تظهر هذه المقالة في وهلة أولى لماذا يمثّل التصنيف الذي قام به فيلومان للأنساق الفلسفية تقدّما في تاريخ فلسفة المعرفة، و } \\
& \text { تجيب في وهلة ثانية على الإحراجات التي عبّر عنها كلّ من بوفراس و آنجال بخصوص التعدّدية الفلسفية التي هي نتيجة يستخرجها } \\
& \text { فيلومان من مبادئ و تعريفات تصنيفه للأنساق الفلسفية. و تتمثّل الأطروحة الأساسية لمذه المقالة في الفرضية التالية : لا تمثّل } \\
& \text { التعدّدية الفلسفية موقفا نجبر على التسليم به إذا ما قبلنا بتصنيف فيلومان. و نقدّم في خاتمة هذا البحث بعض المعطيات التي تشكّل } \\
& \text { برهنة على هذه الفرضية. } \\
& \text { كلمات- مفتاح : فلسفة المنطق، منطق كلاسيكي، منطق حدسي. }
\end{aligned}
$$

Résumé Cet article montre dans un premier temps pourquoi la classification que Vuillemin donne des systèmes philosophiques est un progrès dans l'histoire de la philosophie de la connaissance, et il répond dans un second temps aux embarras exprimés par Bouveresse et Engel au sujet du pluralisme philosophique qui est une conséquence que Vuillemin tire des principes et définitions de sa classification des systèmes philosophiques. La thèse principale de cet article est une conjecture : le pluralisme philosophique $n$ 'est pas une position que l'on est contraint d'admettre si l'on adopte 
la classification de Vuillemin. On donne pour conclure les éléments qui devraient permettre la démonstration de cette conjecture.

Mots-clefs : Philosophie de la logique, logique classique, logique intuitionniste.

(C) 2014 AL-Mukhatabat, $\mathrm{N}^{\circ}$ 11/ Juillet 2014

Joseph Vidal-Rosset : Pluralisme philosophique versus logique intuitionniste, p. 17-197.

Les systèmes philosophiques ont dû produire la dialectique pour s'éprouver les uns les autres et la logique pour s'éprouver eux-mêmes.

Vuillemin

What goes for the laws of logic goes more generally for the principles of philosophy.

Dummett

\section{Objet et but de cet article : Vuillemin versus Dummett}

Je dois reconnaître humblement que mes aînés Bouveresse [2] et Engel [11] ont soulevé plus tôt que moi le problème crucial que la classification de Vuillemin pose par rapport à la question du rapport entre vérité philosophique et vérité tout court. Cet article porte dans un premier temps sur la question du statut de la logique dans la classification des systèmes philosophiques de Vuillemin. Dans un second moment on s'efforce de montrer que, contrairement à ce que Vuillemin soutient, l'entorse principale que Vuillemin fait au concept de vérité ordinaire (à savoir sa thèse du pluralisme philosophique) n'est pas une conséquence nécessaire de l'adoption de sa classification. L'article ne s'achève pas sur une démonstration mais sur une conjecture qui est vraisemblablement démontrable.

Je dédie cet article à Jacques Bouveresse qui plus que quiconque a su montrer l'intérêt et la richesse de la classification de Vuillemin. Comme le montre son livre Qu'est-ce qu'un système philosophique? [3], publié à partir de ses cours au Collègue de France ${ }^{1}$, Bouveresse a vu bien avant moi que, sur la question de la nature systématique de la philosophie, la confrontation des positions respectives de Vuillemin et Dummett est particulièrement éclairante. Convaincu depuis quelques années du caractère fécond de l'usage de la logique intuitionniste dans l'analyse des questions philosophiques, j'ai pris ici le parti de Dummett, en m'efforçant de démontrer que le pluralisme philosophique de Vuillemin n'est pas une thèse qu'un intuitionniste à la Dummett est contraint d'admettre.

D'aucuns trouveront sans doute que la tournure more geometrico de certains passages de cet article a quelque chose d'affecté ou de forcé mais ne suffit pas à prouver que les arguments avancés sont des démonstrations au même titre que des démonstrations de logique

Je remercie vivement Sara Negri, Roy Dyckhoff et Göran Sundholm pour la correspondance très utile qu'ils ont bien voulu prendre le temps d'avoir avec moi, afin de m'éclairer sur le sujet difficile des «plongements » en logique mathématique.

${ }^{1}$ Je cite dans cet article la traduction que Bouveresse donne dans cet ouvrage du livre de Vuillemin, What are Philosophical Systems? [41] qu'il commente dans cet ouvrage. 
mathématique. À cette critique je réponds simplement que j'ai adopté ce style afin de m'efforcer à autant de rigueur et de concision que possible ; compte tenu de la difficulté du sujet, j'espère qu'on ne trouvera pas cette décision inutile.

\section{Les raisons du caractère opératoire de la classification de Vuillemin}

En définissant sa classification des systèmes philosophiques Vuillemin $[\mathbf{4 0 , 4 2 , 4 1 ]}$ a réalisé son chef d'oeuvre, comme le souligne Bouveresse [3, Cours 1] :

La philosophie de la philosophie a pris, chez [Vuillemin], la forme d'une tentative de construction d'une classification et d'une théorie des systèmes philosophiques qui, de toute évidence, a constitué un des aspects les plus importants, pour ne pas dire le plus important, de son oeuvre.

Mis à part le livre de Bouveresse [3] déjà cité, et un volume d'articles en hommage à Vuillemin édité par Rashed et Pellegrin [29], il y a eu, jusqu'à présent, peu de travaux universitaires sur la classification de Vuillemin. Impressionné par les exposés qu'il avait donné de sa classification lors de ses leçons au Collège de France au début des années 80, j’y avais consacré ma thèse de doctorat où je m'efforçais de montrer comment la classification esquissée par Quine $[\mathbf{2 0}, \mathbf{2 1}]$ en philosophie des mathématiques pouvait être intégrée à celle que Vuillemin donne pour les systèmes philosophiques en général. Bouveresse m'a fait un honneur que je ne crois pas mériter, d'une part en citant généreusement ce travail dans son cours du Collège de France, d'autre part en corrigeant avec indulgence et discrétion, la tendance que j'ai eue dans cette thèse à forcer le trait ; il est en effet tout à fait juste de dire comme il le fait que [3, Résumé du cours de l'année 2008]:

Quine n'a pas réellement cherché à construire une classification en bonne et due forme des différentes espèces de philosophies des mathématiques et encore moins, bien entendu, des différentes espèces de philosophies tout court. Il a plutôt cherché, plus modestement, à montrer comment les trois espèces principales de philosophies des mathématiques qui se sont divisées et affrontées au vingtième siècle : le logicisme, l'intuitionnisme et le formalisme, peuvent être distinguées par les engagements ontologiques auxquels elles consentent ou refusent de consentir et comment le critère de l'engagement ontologique qu'il propose permet de clarifier les désaccords qu'il y a entre elles.

En dépit de cette critique pertinente et de toutes les autres que l'on pourrait encore faire sur ce travail de jeunesse que j'aurais du mal à relire, il y a au moins un point que je garde en mémoire comme le résultat des analyses développées dans cette thèse de doctorat : il s'agit de l'adéquation du critère d'engagement ontologique de Quine avec le fil conducteur de la classification de Vuillemin. Rappelons que l'engagement ontologique selon Quine [25, chap. 4, pp. 107-129] se définit avec clarté et distinction dès lors que l'on dispose du langage du calcul des prédicats du premier ordre :

Notre question était la suivante: quels objets requiert une théorie ? Voici notre réponse : ce sont les objets qui ont à être des valeurs de variables pour que la théorie soit vraie. [...]. L'existence est ce qu'exprime la quantification existentielle. Il y a des choses de l'espèce $F$ si et seulement si $\left(\exists_{x}\right) F x$.

Vuillemin [43, pp. 6-7] a confirmé cette adéquation en écrivant : 
Le philosophe se demande, en somme, où placer dans le monde les objets mathématiques ou bien comment concevoir le monde pour qu'ils puissent y trouver leur place. Et, comme l'existence des objets ou places dans le monde correspondent aux formes possibles de prédication en tant qu'elles sont susceptibles de recevoir une valeur de vérité, à l'exclusion donc des fictions, il y aura autant de philosophies [ou plus exactement, de classes de systèmes philosophiques] qu'on peut distinguer de formes de prédication originales. La philosophie des mathématiques, à cet égard, se confond avec la philosophie théorique et l'histoire des classes de systèmes.

Dans la citation qui précède j'ai souligné les points qui établissent l'adéquation entre le critère d'engagement ontologique de Quine et la manière dont Vuillemin caractérise la classe des systèmes dogmatiques. Chauvier [5, pp. 190-191] souligne très justement que le passage de la perception au langage se fait, du point de vue de Vuillemin [41], lorsqu'il est question de « parler d'objets ». Je ne crois pas que Quine [25, chap. 1] soutienne une thèse fondamentalement différente sur cette question. Si l'expression objectuelle de la norme de l'engagement ontologique est insuffisante dès lors qu'il s'agit de traduire le changement de perspective qui caractérise les systèmes qui appartiennent à la classe des systèmes de l'examen, en revanche le critère de Quine offre un gain de simplicité qui permet de comprendre que la classe des systèmes dogmatiques est ordonnée selon un principe d'économie ontologique si l'on part du réalisme des Idées pour aller jusqu'au nominalisme en passant par le conceptualisme.

Lors de son cours au Collège de France, Vuillemin a présenté la progression de l'économie ontologique réalisée sur l'engagement du réalisme des Idées d'une manière très simple que je reconstitue ici de mémoire dans un vocabulaire qui n'est pas toujours le sien.

La formule qui caractérise l'engagement ontologique d'un Platon est la disjonction « toute chose est ou bien une Idée, ou bien l'image d'une Idée ». C'est là le résultat du privilège accordé par le réalisme des idées à la prédication pure, c'est-à-dire aux énoncés prédicatifs qui associent deux universaux (par exemple «l'humilité est une vertu », « huit est un nombre pair »). Les autres énoncés sur les objets ou images du monde sensible se comprendront par participation à cette première forme de prédication. Pour qu'un énoncé comme « $\pi$ est un nombre irrationnel et transcendant » soit vrai, il faut admettre dans le parcours des valeurs des variables de la théorie qui assume la vérité de cet énoncé, l'existence d'entités abstraites, en l'occurrence celle du nombre $\pi$ lui-même.

Aristote rejette non la réalité des Idées mais leur transcendance. L'universel n'est dès lors plus dans les Idées, mais dans les choses sensibles, au titre des Formes ou des qualités abstraites par l'esprit. Schématiquement, dans une théorie conceptualiste comme celle d'Aristote, le parcours de valeurs des variables est composé des individus (substances premières) et des espèces (substances secondes). L'affirmation «toute chose est un individu ou une espèce » exprime le privilège accordé à la prédication substantielle composite (« Socrate est un homme ») et à la prédication accidentelle simple (« Socrate est assis ») caractéristique du conceptualisme. Par rapport au réalisme, le conceptualisme réalise une économie ontologique comparable à celle qu'une théorie prédicative des ensembles réalise par rapport à une théorie imprédicative : on rejette les ensembles qui ne seraient pas définis à l'aide d'éléments auparavant spécifiés. La progression des abstractions est dès lors comparable à celle que Russell [30, Appendice B] a décrite dans sa théorie des types simples. 
Une économie ontologique encore plus sévère est réalisée par les philosophes que l'on désigne par le terme de «nominalistes », parce qu'ils nient que l'universel soit dans les choses et ne le posent que dans les mots ou dans les signes, raison pour laquelle la philosophie des mathématiques contemporaine qui est l'expression du nominalisme a été le formalisme. En ce qui concerne la réalité physique, l'univers de ces philosophes se réduit à l'existence des individus, lorsqu'ils privilégient prédication substantielle simple ( $x$ est un atome ») et prédication accidentelle composite («Jupiter est occulté par la Lune ») ou à celle des événements quand ils privilégient la prédication circonstancielle (« il a plu à Paris le 14 juillet »). La description de la classe des systèmes dogmatiques achevée, on peut faire deux remarques. Premièrement, c'est bien la question de l'existence des objets abstraits et de leur définition dans la description de la réalité qui est le fil conducteur de la classification de Vuillemin. Deuxièmement, comme le remarque Quine [20], celui qui assume l'engagement ontologique le plus faible a la charge de prouver qu'il peut décrire la même réalité (en l'occurrence l'univers des mathématiques) en paraphrasant les assomptions ontologiques des partisans de l'ontologie la plus lourde dans un langage ontologiquement plus faible.

Le choix de Quine en faveur du réalisme en mathématiques est fondé sur le double constat suivant. D'une part il est impossible à un philosophe qui veut rendre compte de l'intégralité de la science mathématique contemporaine de faire l'économie d'une théorie des ensembles imprédicative comparable à celle de Zermelo ; les théories prédicatives (conceptualistes du point de vue de Vuillemin) échouent à traduire des théorèmes importants des mathématiques classiques, comme Quine [23, pp. 249-265] le montre pour le théorème de la borne supérieure de toute partie majorée de l'ensemble des nombres réels. D'autre part, le projet nominaliste qui consiste à tenter de traduire les mathématiques dans un langage ontologiquement neutre comme l'est celui du calcul des prédicats du premier ordre est un projet vain si l'on tombe d'accord avec Frænkel et al. [13, p. 333] pour dire que les difficultés qui existent pour paraphraser toutes les mathématiques classiques en termes nominalistes semblent et probablement sont insurmontables. On voit donc que la norme de l'engagement ontologique a pour mérite d'être un critère objectif pour juger de l'engagement ontologique réel d'une théorie et qu'elle offre aussi un moyen de comparer les théories entre elles. Vuillemin était admiratif de la critique de Quine $[\mathbf{2 4}, \mathbf{2 6}$ ] au sujet des prétentions nominalistes de Russell qui sont désaccord avec la théorie des classes développée dans les Principia. La norme de l'engagement ontologique permet de montrer que cette théorie des classes est en fait une théorie réaliste. Il n'est pas douteux que la classification de Vuillemin exprime le même souci de clarifier la question des ontologies adoptées par les systèmes qu'elle permet de juger. Mais j'ai déjà insisté sur ce point [33].

Cette explication conduit à son tour à une question qui ne peut pas manquer de se poser : ces oppositions ontologiques en philosophie et cette classification ne sont-elles pertinentes que pour la philosophie des mathématiques?

S'il est vrai que pour Vuillemin [40, pp. 288-289] le fait de privilégier un couple d'assertions fondamentales suffit à fonder un système philosophique complet, il n'en reste pas moins vrai qu'il n'exclut pas que l'on puisse par exemple, adopter le réalisme comme philosophie des mathématiques, le conceptualisme comme philosophie de la nature et le nominalisme en morale. Un tel éclectisme est à ses yeux «instable » sur le plan philosophique et, comme je 
l'ai remarqué ailleurs [35], ce souci de stabilité peut aussi être exprimé, comme Tennant l'a fait, du point de vue logique défendu par l'intuitionnisme.

Après avoir réussi à montrer que la philosophie de la connaissance de Bachelard pouvait être définie comme un intuitionnisme au sens que Vuillemin donne à ce mot [32], j'ai été définitivement convaincu du caractère opératoire de cette classification lorsqu'il s'agit de saisir une authentique position philosophique. Mais il est plus difficile d'expliquer les raisons du caractère opératoire de la classification de Vuillemin que de simplement le remarquer. On doit effectivement se poser la question de savoir pourquoi la classification de Vuillemin est fiable dès lors que l'on entreprend d'expliquer par exemple les caractéristiques essentielles du système d'Aristote, les points sur lesquelles il s'oppose à la philosophie de Platon, ou encore ce qui sépare fondamentalement la perspective d'un Descartes de celle d'un Spinoza. Cette question de la fiabilité de la classification de Vuillemin revient à celle de son adéquation avec l'histoire de la philosophie et conduit à se demander si cette classification est complète : comment être certain que des options philosophiques fondamentales n'échappent pas à cette classification ? La réponse à ces questions achèvera la section de cet article où je soutiens qu'au regard de la philosophie de la connaissance la classification de Vuillemin est non seulement à la fois fiable et complète, mais permet de définir une méthode d'identification des différents systèmes philosophiques dans l'histoire de la philosophie.

A la question qui est de savoir si la classification est complète, la réponse de Vuillemin ne fait aucun doute : elle l'est parce qu'elle repose sur une classification complète des formes fondamentales de la prédication et que les systèmes philosophiques reposent sur un privilège accordé à telle ou telle assertion fondamentale ou à tel ou tel couple d'assertions fondamentales pour donner une description intégrale de la réalité. Dans un article publié en 1984, Vuillemin affirme le caractère ontologique et complet de la classification qu'il est en train d'élaborer [39, p. 11 ; c'est moi qui souligne] :

\footnotetext{
Le système ne sera démontré complet que si, dans ce prélude philosophique [qu'est l'exercice classificatoire des formes fondamentales de la prédication], on subordonne la sémiotique à un principe philosophique et on la rend, par là, générale. Quel est ce principe ? Les participants à la communication doivent, pour que la communication puisse réussir, accéder aux conditions de la vérité que, par supposition, vise la prédication. On aura donc classé exhaustivement les formes fondamentales de prédication en décrivant comment les participants à la communication peuvent et doivent, étant donné leurs positions particulières, s'accorder sur ces conditions. La classification sera complète au moment où les participants conviendront que l'introduction d'une forme nouvelle ferait s'évanouir les conditions de vérité, comme on verra qu'il arrive pour la fiction. [. . .] Je distinguerai trois articulations principales de la classification : celle de la forme nominale, celle des formes verbales objectives, celle des formes verbales subjectives.
}

Pour résumer, c'est parce que la classification est fondée sur la série complète des assertions fondamentales permettant la communication de la perception et qu'un système philosophique authentique s'explique par un privilège accordé à telle ou telle assertion fondamentale, que la classification des systèmes philosophiques est nécessairement complète. Identifier la nature d'un système philosophique revient donc à repérer ses principes fondateurs, autrement dit à déterminer ce qu'il privilégie dans la description intégrale de la réalité. Les systèmes philosophiques authentiques étant incompatibles, il en résulte des conséquences importantes qu'il s'agit maintenant d'examiner. 
A juste titre, Bouveresse souligne que Vuillemin n'est pas un philosophe positiviste, si l'on entend par ce terme quelqu'un qui croit qu'un problème philosophique, quel qu'il soit, doit tôt ou tard trouver une solution qui s'impose universellement via une preuve ou une réfutation scientifique purement rationnelle ou expérimentale. Tout au contraire, Vuillemin était parvenu à la conviction qu'un problème authentiquement philosophique trouve une pluralité de solutions rationnelles mais mutuellement incompatibles, chacune fondée sur des argumentations qui ne sont jamais indubitablement décisives. L'aporie de Diodore Kronos n'est évidemment pas aux yeux de Vuillemin le moteur de l'histoire de la philosophie, mais elle est le type même du problème philosophique par excellence, car à son sujet s'exprime un nombre défini de positions philosophiques et des développements indéfinis d'arguments. Un positiviste pensera, peut-être naïvement, que même cette aporie célèbre doit avoir une solution logique qui est préférable à toutes les autres, parce qu'au moins elle l'emporte sur les autres par son caractère plus simple et plus naturel. Vuillemin s'est toujours refusé de croire à la possibilité d'un tel verdict et je l'entends encore me rappeler, lors d'un colloque à Clermont-Ferrand organisé par Elisabeth Schwartz, à quel point il était pluraliste en philosophie, ce qui signifiait qu'il ne croyait pas à un traitement logique définitif et satisfaisant d'une aporie philosophique aussi fondamentale que celle de Diodore. On retrouvera plus loin la question du pluralisme ; cette digression permet simplement de comprendre que le socle de la philosophie pour Vuillemin n'est pas dans la logique, au sens précis que l'on donne à ce mot en mathématiques et en informatique fondamentale, mais dans l'ontologie, dans « l'ontologique soumise à la logique », comme Bouveresse [3, Cours 3] le rappelle en citant et traduisant ce passage de What are Philosophical Systems? [41, p.105]:

Comme tous les systèmes axiomatiques ont en commun l'appareil déductif qui est appelé la logique
formelle, nous pouvons définir le système de signes qui est particulier à la philosophie comme une
ontologie soumise à la logique. Cela étant, il n'est pas étonnant que les parties premières de la
logique - la syllogistique et la logique propositionnelle - aient trouvé leur expression scientifique à
l'intérieur des philosophies grecques. En outre, les deux dimensions qui sont attribuées à la
philosophie comme combinaison présumée cohérente de signes ontologiquement interprétés,
expliquent pourquoi chaque philosophie libre a la forme d'un système rationnel. Puisque la logique
s'applique à l'ontologie, la philosophie est systématique de la même façon que les systèmes
axiomatiques matériels le sont. De plus, un ensemble quelconque de prémisses qui contient une
ontologie continue à embrasser le tout de la réalité, et la philosophie peut être dite être
systématique dans un second sens, qui rappelle et métamorphose l'universalité du mythe.

Je ne crois pas trahir la pensée de Vuillemin en affirmant qu'il y a, dans l'histoire de la philosophie telle qu'il la comprend et en dépit de ce que pourrait faire croire son expression, " l'ontologie soumise à la logique », une priorité de l'ontologie sur la logique, et peut-être même à ses yeux - aussi surprenante que puisse paraître une telle affirmation pour ceux qui imaginent un Vuillemin scientiste - une influence plus forte du mythe que de la science dans l'activité philosophique. Il faut bien sûr justifier ces deux affirmations. En raison de leur caractère crucial dans l'argumentation de cet article, je les distinguerai par les deux propositions suivantes.

Proposition 1.1. - Selon Vuillemin, tout système philosophique se définit à partir d'un choix philosophique qui peut se comprendre comme un choix ontologique; la soumission de tout 
système philosophique à "la logique » n'est qu'une conséquence de la supposition de la cohérence du choix qui définit le système en question. Lorsque certains principes logiques fondamentaux sont assumés ou sont refusés, comme le principe de bivalence ou le tiers exclu, c'est toujours en raison de la cohérence du choix.

Proposition 1.2. - l'expression "la logique " ne désigne dans la classification de Vuillemin rien d'autre que tel ou tel système logique défini en raison d'un choix philosophique [40, p. 285] : "Les systèmes philosophiques ont dî produire la dialectique pour s'éprouver les uns les autres et la logique pour s'éprouver eux-mêmes ». (Sinon le refus d'Épicure d'admettre le tiers exclu comme une vérité logique serait considéré comme absurde du point de vue de la classification, ce qui serait absurde.)

Proposition 1.3. - A la différence du mythe, tout système philosophique admet l'exigence logique de cohérence, et en cela admet un critère reconnu par toute théorie scientifique.

A l'appui de cette proposition, on reprendra la citation que Bouveresse [3, Cours 10] donne de cet alinéa de Nécessité ou contingence [40, p. 285] :

La pluralité des philosophies, leur rivalité, leurs polémiques rappelèrent, dès l'origine, à la raison que poser, c'est se diviser et choisir. Comment la faculté même des principes pouvait-elle produire un tel conflit? Car c'est le sentiment de cette diversité irréconciliable qui distingue la philosophie du mythe. Celui-ci va rapiéçant des bouts, sans s'inquiéter du disparate. Celle-là ne pose un principe qu'au vu de ses conséquences. Si elle n'y prenait pas garde, une autre la rappellerait aussitôt à la cohérence.

Proposition 1.4. - Par définition tout système philosophique authentique est " un système intégral de la réalité » $[\mathbf{4 0}$, p. 286] et, en raison du caractère absolument universel de ce qu'il définit comme la réalité, il hérite d'une caractéristique propre au mythe et se distingue des systèmes scientifiques.

En ce qui concerne les mathématiques, Vuillemin [41, p. 104] remarque que « en tant que système hypothético-déductif, l'axiomatique est [. . . ] complètement étrangère à l'ontologie ». En somme la question de la nature des figures ou des nombres est une question philosophique. Mais la recherche de la distinction entre apparence et réalité est générale à toute l'entreprise philosophique et s'étend donc à toute chose. Si bien qu'à la différence des sciences de la nature, la réalité ou les objets dont traite un système philosophique ne sont pas rigoureusement définis par un protocole expérimental. C'est pourquoi Vuillemin écrit [41, pp. 131-132] :

Cette indétermination est la garantie de l'indépendance relative de la philosophie. Assurément, en vertu de l'origine commune de l'axiomatique et de la philosophie, il y a des échanges réciproques entre les lois scientifiques positives et les conceptions philosophiques correspondantes des lois. Ce n'est pas par un simple hasard que l'empirisme sceptique est une philosophie de physiciens, alors que personne n'était autorisé à entrer dans l'Académie de Platon s'il ne s'y connaissait pas en géométrie. Une philosophie qui n'est plus nourrie par la science décline et se flétrit en scolastique. Néanmoins les lois scientifiques ne déterminent jamais de façon unique les concepts philosophiques. Les systèmes philosophiques et a fortiori les classes de systèmes philosophiques ne sont jamais confrontés directement avec les lois scientifiques comme avec des expériences 
cruciales. En conséquence, aucune découverte scientifique n'est par elle-même en mesure de forcer une décision philosophique.

Vuillemin avait peu de goût pour les slogans ; néanmoins son propos pourrait ici se résumer par un slogan qui serait, selon lui, le mot d'ordre de la pensée philosophique : «ni mythe, ni science ». La philosophie se distingue du mythe parce qu'elle se soumet à la logique ; mais elle n'est pas non plus une science, parce que, comme le remarque Bouveresse [3, Cours 2], contrairement à Dummett ou à Quine [22, § 56, p. 378] et à tous les philosophes que 1'on peut considérer comme appartenant à la tradition analytique au sens large de ce terme, Vuillemin ne croit pas que la philosophie puisse procéder «conformément à des méthodes de recherche admises par tout le monde » et obtenir des résultats «entérinés ou récusés en fonction de critères communément admis ». Si tel était le cas, la philosophie serait à la fois systématique en raison de la structure logique de ses théories, et systématique au second sens que Dummett donne à ce mot, c'est-à-dire en raison de la nature communément admise de ses méthodes de preuves et de réfutation. Comme l'histoire de la philosophie montre que l'activité philosophique ne s'interdit a priori aucun domaine de réflexion, et qu'il est de la nature des systèmes philosophiques de tenter de donner une sorte de tableau intégral de la réalité à partir d'un nombre restreint de principes, il est vain selon Vuillemin d'une part d'imaginer que l'on puisse parvenir à des thèses philosophiques qui soient universellement reconnues comme des vérités, comme c'est le cas en mathématiques et dans les sciences de la nature, et il est d'autre part tout aussi illusoire de penser qu'une des cinq grandes classes de systèmes définis par sa classification (à savoir réalisme des Idées, conceptualisme, nominalisme, intuitionnisme et scepticisme) puisse un jour être définitivement écartée par une théorie scientifique. Les classes de systèmes philosophiques subsistent et subsisteront en raison même de leur origine, c'est-àdire en raison des formes fondamentales de la prédication et du privilège que l'on accorde à certaines d'entre elles quand on prend le risque de penser philosophiquement. Ce point important a une fois de plus été impeccablement exprimé par Bouveresse [3, Cours 9] lorsqu'il écrit :

[Vuillemin] pense que les systèmes philosophiques, pour des raisons qui n'ont rien d'accidentel, sont réellement irréfutables. Ils ne le sont pas simplement du point de vue subjectif de la psychologie de l'adhésion et de la croyance.

Autrement dit le pluralisme philosophique est une conséquence nécessaire aux yeux de Vuillemin des principes mêmes sur lesquels sont fondées sa classification des systèmes. Le pluralisme philosophique de Vuillemin n'est en rien une posture morale, je veux dire qu'il n'est pas une concession faite à un esprit du temps où il serait de bon ton d'être pluraliste comme il politiquement correct d'être démocrate. Il est certain en revanche qu'il a, aux yeux de Vuillemin, au moins une conséquence morale, comparable à celle de la théorie kantienne qui met le philosophe à l'abri de la Schwärmerei. L'agnosticisme du pluralisme est moralement préférable au dogmatisme vulgaire et fanatique. Mais ce ne sont certainement pour ces raisons morales que Vuillemin considère que le pluralisme s'impose, il s'impose à l'esprit du philosophe de la connaissance qu'il est, pour des raisons théoriques, celles qui tiennent à l'élaboration de la classification, et par fidélité à l'histoire de la philosophie qui témoigne de la survivance des ces classes de systèmes, quelle que soit l'actualité scientifique. 
Voilà ce que Vuillemin soutient lorsqu'il écrit [41, p. 132] que « les philosophies sont vivantes parce qu'elles peuvent être indéfiniment réécrites ».

On ne peut être, à mon avis, que profondément admiratif de la lumière que l'œuvre classificatrice de Vuillemin jette sur la nature et l'histoire de la philosophie. Je crois me souvenir avoir entendu Vuillemin au Collège de France, soutenir qu'il y avait aussi progrès dans les sciences lorsqu'une théorie parvenait à être exposée et expliquée de façon suffisamment simple et claire pour des générations d'étudiants. Je me souviens, avec certitude cette fois, l'avoir entendu dire qu'il ne cherchait pas à développer une philosophie difficile et compliquée mais simple, claire et distincte. Lors de l'exposé que Vuillemin fit de sa classification au Collège de France, je garde en mémoire le vif souvenir du sentiment de lumière jetée sur les polémiques philosophiques que j'étudiais jusqu'alors sans ordre, c'est-àdire sans en saisir ni la nature, ni l'unité. Je ne crois pas avoir connu depuis d'expérience comparable à cette joie de comprendre enfin le caractère fondamentalement polémique de la philosophie et ses principaux conflits. Plus tard, j'ai pu comme enseignant mettre à profit ce que j'avais appris, et grâce à cette oeuvre magistrale, je pense avoir orienté correctement la pensée de mes étudiants en leur apprenant que, comprendre un système philosophique, c'est avant tout saisir les principes fondamentaux de sa constitution ainsi que sa portée polémique par rapport aux autres systèmes qu'il affronte. En un mot, je n'imagine pas comment il est possible de comprendre la nature de la philosophie et de son histoire sans faire référence à l'oeuvre de Vuillemin. La section suivante, qui est une critique à la fois de son pluralisme philosophique et de son platonisme est encore une façon de lui donner raison sur la thèse du caractère foncièrement polémique de la philosophie.

\section{Le pluralisme philosophique et l'argument de la charge de la preuve}

La conjecture selon laquelle le pluralisme philosophique ne serait pas une conséquence que l'on doit nécessairement admettre si l'on adopte la classification de Vuillemin ne doit pas être conçue comme une réfutation du pluralisme philosophique, car il faudrait pouvoir montrer que la thèse du pluralisme philosophique est absurde, or il est évident qu'elle ne l'est pas. Il ne faut pas non plus entendre par «pluralisme philosophique » la platitude qui consiste à reconnaître qu'il existe une pluralité de positions philosophiques inconciliables. Le pluralisme philosophique affirme quelque chose de bien plus précis ; il trouve une de ses expressions dans cette formule de Vuillemin :

Proposition 2.1. - Aucune découverte scientifique n'est par elle-même en mesure de forcer une décision philosophique.

Une telle assertion est déjà éminemment problématique lorsqu'on la considère hors de son contexte, c'est-à-dire indépendamment des principes de la classification qui selon Vuillemin, l'entraînent. Dès lors que l'on n'adopte pas le pyrrhonisme et que l'on accepte la thèse selon laquelle la philosophie a comme point commun avec la science la poursuite de la vérité, on voit mal les raisons pour lesquelles une découverte scientifique serait par elle même incapable de forcer une décision. Il est nécessaire d'admettre, même du point de vue qui est celui de Vuillemin, qu'il y a des arguments logiques qui réfutent certaines interprétations 
philosophiques. J'ai fait allusion plus haut à l'argument de Quine [24] selon lequel la théorie russellienne des classes est contrainte de reconnaître l'existence des objets abstraits que sont les ensembles et donc exprime un engagement ontologique réaliste. Vuillemin considérait cet argument de Quine comme la démonstration d'une vérité importante dans l'histoire de la philosophie contemporaine. De même il louait Quine d'avoir montré avec précision toutes les difficultés conceptuelles qu'implique une position rigoureusement nominaliste.

Aucune de ces remarques n'aurait été cependant susceptible d'inquiéter Vuillemin sur la cohérence de sa philosophie de la philosophie. En ce qui concerne l'argumentation de Quine contre le nominalisme, il aurait probablement répondu que le nominalisme peut retrouver d'autres formes d'expression et détourner les difficultés logiques que cette position rencontre en philosophie des mathématiques. Enfin Vuillemin aurait sans difficulté reconnu qu'il existe dans l'histoire de la philosophie des arguments décisifs qui prouvent ou réfutent des thèses que l'on peut isoler, que l'on peut considérer comme locales, mais qui en aucun cas ne sont comparables aux thèses fondamentales qui sont les principes des classes de systèmes philosophiques. Quine a réfuté la thèse selon laquelle les Principia Mathematica développent une théorie nominaliste des classes ; il n'a pas réfuté le nominalisme en général. Lorsque Vuillemin [43, p. 38] soutient que « la philosophie ne comporte pas de démonstration », il prend soin de préciser qu'il ne veut pas dire par là qu'il n'y a pas de critère de décision rationnel en philosophie, mais plus simplement, et plus fondamentalement, qu'il n'est possible ni de prouver la vérité de la base théorique d'une position philosophique (c'est-à-dire démontrer la vérités des principes de la classe à laquelle appartiennent tel et tel système philosophique), ni de réfuter une classe de système philosophique.

L'adoption du pluralisme entraîne chez Vuillemin une compréhension très précise de ce qu'est non seulement un choix philosophique mais aussi une dispute philosophique. Un choix philosophique se fait, sous la contrainte de la logique, avec la reconnaissance de ne pouvoir à partir du point de vue adopté justifier des positions que l'on reconnaît comme logiquement possibles. Puisque dans le traitement d'une aporie comme celle de Diodore, le philosophe fait librement un choix cohérent, il reconnaît d'une part s'écarter du sens commun insouciant de la cohérence de la description de la réalité et, d'autre part, ne pas adhérer à certaines autres positions philosophiques possibles.

C'est pourquoi Vuillemin [41, p. 133] écrit :

Une classification philosophique, si elle est utile, jette une lumière sur notre situation en relation aux autres, en nous rappelant qu'il faut reconnaître qu'eux aussi ont de bonnes raisons pour choisir conformément à une maxime qui n'est pas la nôtre.

Je me souviens avoir entendu Vuillemin définir dans un de ses cours, les systèmes philosophiques, comme " des systèmes d'amputation », voulant dire par là que, sous la contrainte de la cohérence, un choix philosophique conduit à sacrifier au moins une assertion reconnue comme allant de soi par la pensée commune non critique, comme par exemple « il existe au moins un événement possible qui n'est pas actuellement vrai et qui ne le sera jamais», et qui est également admis par des systèmes philosophiques différents. Mais si l'on admet qu'un philosophe ne peut ni démontrer la vérité des principes qui sont les siens, ni réfuter ceux qu'il rejette, qu'est-ce qu'un argument philosophique ? Vuillemin n'a hélas pas eu le temps de répondre de manière développée à cette question, mais il était parvenu à la 
conclusion que l'argument philosophique par excellence s'apparente à l'argument juridique de la charge de la preuve. On va maintenant expliquer dans un premier temps ce que signifie cet argument juridique, et dans un second temps les raisons pour lesquelles Vuillemin considère que cet argument est le type même de l'argument philosophique.

En droit civil, la règle générale est que la charge de la preuve incombe au plaignant, et que celui qui ne porte pas la charge de la preuve a du même coup le bénéfice de la présomption d'innocence. Bien évidemment, c'est à un juge extérieur au conflit que revient la responsabilité de dire qui a la charge de la preuve et qui jouit de la présomption d'innocence. D'un point de vue scientifique, on peut aisément comprendre que la charge de la preuve incombe à celui qui soutient des assertions, que celles-ci soient de nature empiriques ou formelles. Prouver un énoncé universel revient ou bien à en donner la démonstration formelle, ou bien montrer que cet énoncé universel permet d'exprimer une régularité naturelle. On peut aussi réfuter un énoncé universel en donnant la preuve de l'existence d'un contre-exemple, tout comme on peut admettre que l'on prouve un énoncé existentiel en exhibant un exemple. Il est cependant inutile ici de poursuivre car l'on voit que, dès lors que l'on s'interroge sur le concept de la charge de la preuve en sciences, on a inévitablement tendance à se poser la question « qu'est-ce qu'une preuve ? », ce qui évidemment n'est plus tout à fait le même sujet. L'analyse de l'argument de la charge de la preuve est importante en philosophie car, comme le remarque Walton [44, p. 233], bien que 1'on on sache depuis Socrate que la dispute théorique entre deux protagonistes est une forme classique de l'argumentation philosophique, tout ce qui a été publié sur le rôle du poids de la preuve en philosophie a conduit à plus de questions que de réponses sur ce sujet. Presque dix années plus tard, Cargile [4, p. 69] non seulement ne contredit pas cette remarque, mais l'appuie en citant Lehrer [15] qui affirme que, «généralement, les arguments qui portent sur la question de savoir à qui incombe le poids de la preuve ne sont pas concluants ».

Pourtant 1'article de Walton s'efforce de clarifier la question, puisqu'il ne s'interroge pas uniquement sur les usages fallacieux de l'argument du poids de la preuve, mais aussi sur le rapport du poids de la preuve et de la plausibilité, se demande si le poids de la preuve revient à celui qui attaque une thèse ou à celui qui la défend, et s'interroge enfin sur les différences entre usage juridique et usage philosophique de cet argument. Il est assez facile de comprendre que le croyant ne peut pas rationnellement faire reposer la preuve de l'existence de Dieu sur le fait que les athées n'ont pas démontré rationnellement son inexistence. De la même façon, pour prouver que tous les chiffres apparaissent avec la même fréquence dans le développement décimal de $\pi$, il ne suffit pas de dire qu'on a toujours échoué jusqu'à présent à montrer un nombre qui n'a pas cette propriété d'apparaître avec la même fréquence que tous les autres dans ce développement décimal. Ces points de logique sont importants mais ils ne font que montrer les usages fallacieux de l'argument du poids de la preuve, sans dire quel est son usage correct dans une dispute philosophique.

Dans la partie positive de son article, Walton semble vouloir s'appuyer, si je le comprends bien, sur le paradigme des jeux ou des dialogues tel qu'il a été développé par Hintikka et Lorenzen. Mais, s'il s'agit de comprendre l'usage que Vuillemin fait de cette forme d'argument, la référence de Walton à la méthode des jeux ou du dialogue en logique est insuffisante pour deux raisons. La première est que l'on peut montrer, comme je l'ai fait avec Galmiche et Larchey-Wendling [34], que la dialogique est une méthode de logique que l'on 
peut toujours traduire par une autre, comme celle du calcul des séquents par exemple, et que les preuves données par la dialogique sont des preuves logiques tout court. Le jeu de dialogue n'est donc ni un vrai jeu, puisque l'issue est déterminée, ni un vrai dialogue, puisqu'on pourrait le convertir en monologue et ne rien perdre du résultat. Pour comprendre l'argument du poids de la preuve tel que le conçoit Vuillemin, la logique ne suffit pas, il est nécessaire d'admettre au moins deux philosophes qui admettent des ontologies rivales et incompatibles pour décrire une même réalité. Dans la perspective de Vuillemin, la proposition qui suit définit l'usage légitime de l'argument de la charge de la preuve.

Proposition 2.2. - Soit deux philosophes $A$ et $B$ qui, pour décrire une même réalité $R$, soutiennent deux théories différentes et incompatibles, respectivement $T_{A}$ et $T_{B}$ qui peuvent être par exemple représentées par les formules suivantes :

$$
T_{A}=\{\exists x F x \wedge \exists y G y\}
$$

et

$$
T_{B}=\{\neg \exists x F x \wedge \exists y G y\}
$$

Si B soutient une ontologie moins lourde que A, comme c'est par exemple ici le cas, alors c'est à $B$ qu'incombe la charge de prouver que l'on peut se dispenser d'assumer l'existence de certaines entités que A admet (en l'occurrence les entités qui sont des $F$ ) pour donner un modèle de $R$.

Le privilège accordé par Vuillemin à ce type d'argument peut s'expliquer par le fait que Vuillemin a très probablement été influencé, plus qu'il ne l'a explicitement reconnu, par la manière dont Quine a défendu le réalisme mathématique. Quine renonce à la philosophie nominaliste pour laquelle il inclinait spontanément, parce qu'il a réalisé qu'il est indispensable d'admettre la théorie classique des ensembles, et donc de quantifier sur les objets abstraits, pour éviter de sacrifier un bon nombre de vérités mathématiques importantes dans la science contemporaine. Son conservatisme en logique est fondé sur la même maxime de mutilation minimum, « il faut éviter de faire chavirer le navire ». La façon dont Vuillemin [43] s'exprime est évidemment à l'opposé de ce que ce que l'on pourrait appeler « le pragmatisme logicotechnique » d'un Quine qui avoue «poser les objets abstraits au total à contre coeur » [27, II, $\S 11$, p. 56], quand Vuillemin [43, p. 28] par contraste souligne en gras dans le texte que son Credo réaliste «porte sur un article unique qui résume une philosophie des mathématiques : Il existe un monde intelligible »; mais l'usage que Quine et Vuillemin font tous deux de l'argument de la charge de la preuve est rigoureusement le même. Quine attend que les mathématiciens qui réfléchissent sur les théories prédicatives des ensembles montrent qu'il est possible de fonder les théories physiques sur un édifice mathématique ontologiquement moins lourd, mais en attendant il déclare le réalisme mathématique comme la seule option raisonnable à ses yeux pour la philosophie des sciences. Vuillemin opte tardivement mais plus résolument pour le platonisme et défie tous les anti-platoniciens de 
rendre compte intégralement de la science et de la morale sans admettre les Idées de Platon ; à eux la charge de la preuve. ${ }^{1}$

Précisons enfin que, si le privilège de l'argument de la charge de la preuve est une des conséquences du pluralisme philosophique, une des conditions pour la bonne expression du pluralisme philosophique est aussi le choix de la stabilité du système ou de l'authenticité de la philosophie adoptée :

Proposition 2.3. - Un système philosophique est authentique si et seulement si il appartient intégralement à l'une des cinq classes de la classification des systèmes. (Par exemple, s'il est stable ou authentique, un tel système ne peut pas être platonicien pour la philosophie de la connaissance, et nominaliste en morale.)

Proposition 2.4. - D'un point de vue authentiquement philosophique, il est impossible de ne pas admettre que certaines positions philosophiques sont abandonnées en raison de la position philosophique que l'on choisit.

\section{Une conjecture : l'intuitionniste peut s'abstenir d'assumer le pluralisme philosophique}

Comme je l'ai souligné dans l'introduction de cet article, la dernière section de cet article s'efforce de donner des arguments pour prouver une conjecture dont l'intuition est à mon avis présente dans les propos de Bouveresse [3, Cours 2] que je comprends ainsi : il n'y a pas de différence fondamentale entre la division tracée par Dummett entre réalisme sémantique et anti-réalisme sémantique et celle établie par Vuillemin entre systèmes dogmatiques et systèmes de l'examen, et cependant Dummett n'est pas conduit à soutenir comme Vuillemin que le pluralisme philosophique doit nécessairement être admis. Encore une fois, le rejet intuitionniste du pluralisme philosophique n'est pas la négation de l'existence d'une pluralité de positions philosophiques, car cette négation serait absurde. L'intuitionniste rejette la proposition 2.1 en soutenant que la logique intuitionniste est une découverte scientifique capable de forcer le choix de l'intuitionnisme à partir de la connaissance de la classification de Vuillemin et de l'exigence philosophique de construction d'un système intégral. Il s'agit par conséquent de démontrer la conjecture suivante :

Conjecture 3.1. - Le pluralisme philosophique n'est pas une conséquence nécessaire des principes de la classification des systèmes philosophiques de Vuillemin.

\footnotetext{
${ }^{1}$ Le fait que la charge de la preuve soit devenue pour Vuillemin l'argument par excellence du réalisme est attesté par ces lignes d'unmanuscrit inédit [38] sur lequel travaille David Thomasette, doctorant à l'Université de Lorraine :

Discipline commune à tout aveu philosophique, la règle respective à l'usage du poids de la preuve est aussi la règle unique du réalisme. Tous les autres aveux philosophiques la complètent par une règle de leur cru. Que cette dernière ait pour objet l'abstraction, le langage, la déduction ou la simple réflexion, elle assigne à l'aveu une méthode spécifique capable de distinguer la philosophie parmi toutes les autres disciplines, quoique ce soit au moyen d'une marque polémique et donc contestable.

De même, la Préface de ce même manuscrit fait écho au texte cité plus haut : Je crois au monde intelligible :

Cette profession de foi est une proposition philosophique. On verra qu'elle en particularise une autre : toute proposition, pour peu qu'elle soit philosophique, exprime un acte de foi ou d'incrédulité.
} 
Trame de la démonstration. - Il faut entendre par «pluralisme philosophique » le fait de reconnaître que les significations respectives des autres options philosophiques fondamentales ne peuvent pas être correctement exprimées à partir du système adopté. Le pluralisme philosophique en ce sens précis revient à affirmer que la conjonction de la connaissance de la classification et le choix d'un système implique le fait d'admettre que "l'amputation » des positions adverses est inévitable, quelle que soit la classe de systèmes philosophique dans laquelle on se place. Le sens de l'expression « pluralisme philosophique » étant donnée, voici maintenant la trame d'une démonstration possible de la conjecture 3.1.

Le choix de l'intuitionnisme implique le choix de la logique intuitionniste, tandis que le choix du platonisme implique l'adoption de la logique classique. En raison des rapports entre ces deux logiques, il y a deux sens que l'on peut donner au mot d' « amputation » dans ce contexte. Par souci de simplicité, on se place uniquement au niveau du calcul propositionnel pour distinguer amputation extensionnelle et amputation intensionnelle :

- Tous les théorèmes intuitionnistes étant aussi des théorèmes en logique classique, mais non l'inverse, la logique intuitionniste est amputée de certains théorèmes classiques dont le tiers exclu est l'exemple le plus connu. Le choix de la logique intuitionniste comme logique de base implique donc une amputation extensionnelle de certains théorèmes : le fait d'assumer certains énoncés ne préserve pas les conséquences admises en logique classique (par exemple $\neg \neg A \nvdash_{i} A$ ). - L'existence d'un plongement de la logique propositionnelle classique dans la logique propositionnelle intuitionniste implique, comme le montre avec précision Epstein [12, Chap. $\mathrm{X}$, pp. 374-400] qu'il n'existe pas de traduction grammaticale de la logique propositionnelle intuitionniste (IPL) dans la logique propositionnelle classique (CPL). En ce sens là, l'« amputation » relève du domaine des significations : les contre-modèles construits dans IPL pour montrer que certains théorèmes de CPL ne sont pas prouvables en logique intuitionniste, n'ont aucune signification du point de vue classique. ${ }^{1}$ Le choix de la logique classique comme logique de base du système philosophique adopté (par exemple le platonisme) implique donc une amputation intensionnelle de la logique intuitionniste. A partir de cette distinction entre amputation extensionnelle et amputation intensionnelle, la preuve de la conjecture 3.1 pourrait reposer sur l'argument suivant.

Il n'est pas difficile de montrer que, si l'on en reste au niveau de la logique propositionnelle, le platonicien est contraint de reconnaître qu'il procède, comme Quine [22] à des amputations intensionnelles. Toujours au niveau de la logique propositionnelle, l'intuitionniste sera au contraire en mesure de nier qu'il procède à des amputations ontologiques, en affirmant qu'il ne fait qu'adopter une logique qui offre un plus grand pouvoir d'expression que la logique classique et qui exige plus d'informations que celles qu'apportent les preuves non constructives. En effet si A est décidable la formule

\footnotetext{
${ }^{1}$ Ce point devient totalement évident lorsqu'on étudie la méthode des arbres de réfutation en logique intuitionniste exposée par Bell et alii [1] : le contre-modèle opposé à la formule du tiers exclu n'est pas lisible en logique classique, alors qu'on peut, du point de vue intuitionniste, retrouver le raisonnement classique.
} 
AL-MUKHATABAT ISSN 1737-6432 Numéro - issue 11 /July - Juillet 2014

$$
\neg \neg A \rightarrow A
$$

redevient un théorème intuitionniste, et ne signifie alors dans ce cas rien d'autre que

$$
\vdash_{i} \neg \neg \top \rightarrow T
$$

où $T$ est la constante du vrai. Supposons enfin qu'il soit possible à l'intuitionniste d'exprimer à partir de sa logique toutes les positions philosophiques de la classification sans jamais renoncer à la sienne, alors l'intuitionniste ne serait pas contraint de reconnaître que son système est un « système d'amputations ontologiques », puisqu'il ne reconnaît comme existant que les choses au sujet desquelles on a des preuves d'existence, donc le pluralisme philosophique ne serait pas une conséquence nécessaire de la classification de Vuillemin. Tel est le fil conducteur des propositions qui suivent.

\section{Définition 3.2 (Principe de bivalence ou principe de détermination des valeurs de vérité des énoncés)}

Tout énoncé déclaratif est vrai ou faux de manière déterminée, indépendamment de toute procédure de décision.

Définition 3.3. — L'intuitionnisme se définit par le refus d'assumer le principe de bivalence.

Remarque 3.4. - Cette définition est compatible avec le fait de définir l'intuitionnisme comme le fait Vuillemin, à partir d'un privilège donné aux jugements de méthode, mais seulement si par «jugement de méthode » on entend le fait de définir nécessairement la vérité de tout énoncé par sa preuve, ou sa fausseté par sa réfutation, et que l'on refuse d'appliquer le principe de bivalence aux énoncés qui ne sont ni prouvés ni réfutés.

Proposition 3.5. - Certains choix philosophiques impliquent l'adoption ou le rejet de certaines règles logiques, c'est-à-dire des règles d'inférence qui sont déclarées correctes dans tous les contextes.

Première preuve : le cas du platonisme. - Le platonisme peut aussi se définir comme l'a fait Dummett $[8,7,9]^{1}$ par l'adoption sans restriction tous les théorèmes de la logique classique et notamment, pour reprendre la terminologie et la notation de David et alii [28, p. 148] par le fait d'assumer comme loi logique universelle la règle dite "d'absurdité classique ", qui affirme que si, via le contexte d'une théorie $\Gamma$ on peut dériver une contradiction de la négation d'une proposition $A$, alors on peut dériver $A$ de $\Gamma$, ce qui s'exprime par la formule :

\footnotetext{
${ }^{1}$ On peut lire sous la plume de Dummett [7, p.154] :

Despite Hilbert's act of faith that every mathematical problem is solvable, there can be for the platonist no intrisic connection between truth and intuitive provability - the latter implies the former, but not conversly.

From a platonist standpoint, the fact that every mathematical statement is either true or false need not imply that every statement is either provable or refutable.
} 


$$
\frac{\Gamma, \neg A \vdash \perp}{\Gamma \vdash A} \perp_{c}
$$

Cette règle est une conséquence du principe de bivalence : si l'on admet que tous les énoncés sont, de manière déterminée, vrai ou bien faux, alors s'il est faux que l'énoncé $A$ est faux, alors il n'y a pas d'autre possibilité que d'en déduire que $A$ est vrai. Le platonisme admet l'existence de vérités intelligibles qui transcendent la connaissance, donc l'existence d'énoncés qui sont vrais ou faux de manière déterminée et indépendamment de toute procédure de décision. Donc le platonisme admet le principe de bivalence et, par conséquent, il se définit aussi par le fait d'admettre que le tiers exclu et la règle d'absurdité classique sont des règles logiques (c'est-à-dire absolument et universellement nécessaires).

Seconde preuve : le cas de l'intuitionnisme. - L'intuitionnisme se définit par le refus d'assumer le principe de bivalence et donc par le refus d'assumer la règle d'absurdité classique comme règle logique. (En raison de la définition 3.3, de la remarque 3.4 et de la fin de la preuve précédente.)

Proposition 3.6. - Définir l'intuitionnisme à partir d'un privilège donné " jugements de méthode " en raison de la reconnaissance de l'activité du sujet qui accède à la vérité, est une définition correcte de l'intuitionnisme, mais insuffisante d'un point de vue logique, car en l'absence de la définition 3.3, la classification de Vuillemin permet de définir un intuitionnisme instable.

Démonstration. - Admettons qu'un système philosophique intuitionniste, pour prouver certaines de ses thèses, accepte l'usage de la règle d'absurdité classique alors, en raison de la proposition 3.5 un tel système philosophique serait instable au sens que Vuillemin [40, pp. 288-289] donne à cette expression.

Proposition 3.7. - Les désaccords logiques entre les logiciens classiques et les intuitionnistes, apparaissent au sujet de l'ensemble de tous les énoncés qui sont prouvables en logique classique mais ne le sont pas en logique intuitionniste. Appelons ce terrain de la discorde «l'ensemble $\delta »$.

Explication. - Assumer le principe de bivalence revient à accepter que la négation soit involutive, ce qui signifie que son application à elle-même via l'affirmation de la formule $\neg \neg A$ est équivalente à l'affirmation de $A$. En logique intuitionniste la négation de $A$ signifie uniquement que l'assomption de $A$ implique une contradiction, par conséquent $\neg \neg A$ signifie l'absurdité de l'absurdité de $A$, autrement dit la cohérence de $A$. Le fait qu'un énoncé (ou une théorie) soit cohérent n'implique pas qu'il soit prouvé, donc

$$
\nvdash_{i} \neg \neg A \rightarrow A \quad 1
$$

\footnotetext{
${ }^{1}$ Soit $\gamma$ l'ensemble des connecteurs du calcul propositionnel : $\{\neg, \wedge, \vee, \in\}$. On peut démontrer en logique classique que $\{\neg, \vee\}$ et $\{\neg, \wedge\}$ sont des systèmes complets minimaux de connecteurs [6, pp. 53-55], ce qui veut dire que le calcul propositionnel classique pourrait faire l'économie de l'implication matérielle et de la disjonction (resp. de la conjonction) et ne rejeter aucun théorème classique. On démontre en revanche qu'en logique intuitionniste la taille de $\gamma$ est irréductible, ce qui signifie
} 
Que $(A \vee \neg A)$ redevienne une formule prouvable en logique intuitionniste lorsqu'elle est précédée de la double négation, ainsi que toutes les formules de l'ensemble $\delta$, signifie que la cohérence de ces formules logiques de $\delta$ est prouvable. Il n'y a donc pas de réfutation possible d'une quelconque formule $A$ de $\delta$ si par « réfutation » on entend la preuve que la supposition de $A$ implique l'absurde. Telle est la leçon philosophique du théorème de Glivenko [14] : il est absurde de tenter de démontrer l'absurdité des formules de $\delta$.

La logique intuitionniste montre en revanche que toute formule de $\delta$ est telle qu'il est toujours possible de lui opposer un contre-exemple issu d'une théorie indécidable. En effet, si une formule du calcul propositionnel appartient à $\delta$ alors il faut supposer la décidabilité d'au moins un de ses atomes pour qu'elle soit démontrable d'un point de vue intuitionniste. Telle est la leçon philosophique du théorème de von Plato $[37,19]$ dont la démonstration prouve qu'une formule $C$ est dérivable en logique classique si et seulement si la formule

$$
\left(P_{1} \vee \neg P_{1}\right) \wedge \cdots \wedge\left(P_{n} \vee \neg P_{n}\right) \rightarrow C
$$

(où $P 1 \ldots P n$ sont tous les atomes de $C$ ) est dérivable en logique intuitionniste. La critique intuitionniste à l'égard de la philosophie sous-jacente à la logique classique est donc parfaitement fondée : la logique classique présuppose l'omniscience.

Proposition 3.8. - Les disputes philosophiques en logique peuvent s'exprimer à partir de l'analyse des théorèmes de "plongement".

Explication. - Contre le choix de la logique classique comme logique de base, l'intuitionniste peut légitimement soutenir que le choix de la logique classique rend impossible le respect de la signification naturelle de chaque connecteur.

A partir de cet argument, le plongement de CPL dans IPL est un point en faveur de l'intuitionnisme, parce qu'il n'existe pas, en calcul propositionnel, de traduction grammaticale de la logique intuitionniste dans la logique classique, alors que l'inverse est vrai. Pour être capable de donner une interprétation de la logique intuitionniste dans son langage, le logicien classique doit au moins faire usage de la logique modale, en raison du plongement, démontré par McKinsey et Tarski [17], d'IPL dans S4. A partir de ce résultat, un plongement du calcul des prédicats intuitionniste dans le calcul des prédicats classique a été démontré par Motohashi [18], mais il serait erroné d'affirmer que cette démonstration donne le dernier mot à la logique classique. En effet, en 1976 Löb [16] démontre qu'il existe un plongement de la logique classique du premier ordre dans la logique propositionnelle intuitionniste du second ordre, ce qui a été développé et interprété récemment par Sørensen et Urzyczyn [31]. Plus récemment encore Dyckhoff et Negri [10] ont donné une preuve constructive et directe du plongement d'IPL dans S4 que McKinsey et Tarski avaient démontré de manière non constructive.

Si l'on peut montrer qu'existe un plongement d'une théorie $S$ dans une théorie $T$, alors $T$ a certainement une procédure de décision au moins aussi complexe que $S$, mais aussi toute interprétation d'une formule $A$ de $S$ détermine une interprétation de $A$ dans $T$. Enfin, la preuve de Dyckhoff et Negri montre clairement que les intuitionnistes interprètent toute preuve non

qu'aucun connecteur propositionnel n'est traduisible à partir des trois autres [1, p. 211]. Sur l'importance que l'intuitionniste peut accorder à la traduction intuitive des connecteurs dans sa dispute avec le logicien classique, voir [36]. 
constructive comme une requête d'informations supplémentaires que seules peuvent donner les preuves constructives, point que même un platonicien est contraint de concéder. La dispute philosophique sur la question de savoir quelle est la logique de base de la connaissance scientifique pourrait donc espérer trouver une issue si l'on parvenait à définir une logique d'ordre supérieur $T$ au sein de laquelle tous les plongements sont fiables et permettent toujours d'interpréter dans $T$, les formules logiques des théories existantes. En raison du fait que l'algèbre de Boole est un cas particulier de l'algèbre de Heyting, il semble raisonnable de conjecturer que, s'il est possible de définir cette logique d'ordre supérieur, celle-ci est intuitionniste.

Conjecture 3.9. - Il est possible d'envisager l'expression adéquate des classes de systèmes philosophiques telles qu'elles sont définies par Vuillemin en partant de la logique intuitionniste comme logique de base.

Argument en faveur de cette conjecture. - La logique intuitionniste est la seule logique qui permet d'exprimer la division bipartite de la classification de Vuillemin en systèmes dogmatiques et systèmes de l'examen. De ce point de vue logique, les systèmes dogmatiques sont tous ceux qui admettent la logique classique comme logique de base ; quant au scepticisme il peut se définir comme la philosophie qui, contrairement à l'intuitionnisme, nie qu'il soit possible de parvenir à une vérité objective et donc refuse, de manière plus ou moins radicale, de tirer des conséquences ontologiques à partir de preuves effectuées par un sujet pensant. Il est remarquable que cette division bipartite ne pourrait être exprimée au niveau propositionnel, ni par tous ceux qui adoptent la logique classique comme logique de base, ni par un sceptique qui serait nécessairement conduit à déplacer la ligne de démarcation entre systèmes dogmatiques et systèmes de l'examen, car comme l'a dit Vuillemin lors d'un cours au Collège de France que je cite de mémoire, «pour un sceptique, un intuitionniste reste un dogmatique ». En ce sens là, de manière informelle on peut dire qu'il est très probablement possible de montrer l'existence d'un «plongement» de la classification de Vuillemin dans la logique intuitionniste, au sens où celle-ci pourraittraduire de manière fiable celle-là.

Conjecture 3.10. - L'intuitionniste n'est ni contraint de reconnaître la vérité du pluralisme philosophique, ni contraint de concevoir l'argument de la charge de la preuve (au sens juridique) comme le type même de l'argument philosophique.

Argument. - Si la conjecture précédente est démontrable, cette dernière avec la conjecture 3.1 l'est aussi. Il serait alors justifié de refuser d'admettre la thèse de Vuillemin [43, p. 38] selon laquelle « la philosophie ne comporte pas de démonstration ». On privilégierait enfin l'usage scientifique de l'argument de la charge de la preuve sur son usage juridique.

\section{Références}

[1] BELL, J.L. \& DEVIDI, D. \& SOLOMON, G. - Logical Options : An Introduction to Classical and Alternative Logics, Broadview Press, Peterborough, Ontario, Canada, 2001. 
AL-MUKHATABAT ISSN 1737-6432 Numéro - issue 11 /July - Juillet 2014

[2] BOUVERESSE, J. - " Jules Vuillemin entre l'intuitionnisme et le réalisme », in Rashed, R. \& Pellegrin, P. [29], p. 45-79.

[3], Qu'est-ce qu'un système philosophique ? Cours du Collège de France - 2007-2008, La philosophie de la connaissance au Collège de France, revues.org, http://www.revues. org, 2012.

[4] CARGILE, J. - «On the Burden of Proof », Philosophy 72 (1997), no. 279, p. 59-83.

[5] CHAUVIER, S. - « La philosophie de la classification des systèmes philosophiques : criticisme et décisionisme », in Rashed, R. \& Pellegrin, P. [29], p. 187-204.

[6] CORI, R. \& LASCAR, D. - Logique mathématique, vol. 1, Calcul propositionnel, algèbres de Boole, calcul de prédicats, Masson, Paris, 1993.

[7] DUMMETT, D. - « Realism », [9], p. 145-165.

[8] DUMMETT, M. - «Platonism », [9], p. pp. 202-214.

[9] , Truth and other enigmas, Harvard University Press, Cambridge, Mass., 1978.

[10] DYCKHOFF, R. \& NEGRI, S. - « Proof analysis in intermediate logics », Arch. Math. Log. 51 (2012), no. 1-2, p. 71-92.

[11] ENGEL, P. - « Jules Vuillemin, les systèmes philosophiques et la vérité », in Rashed, R. \& Pellegrin, P. [29], p. 29-43.

[12] EPSTEIN, R.L. - Propositional Logics - The Semantic Foundations of Logic, Wadsworth, Thomson Learning, Stamford, USA; London, UK, 2001.

[13] FRÆNKEL, A. \& BAR-HILLEL, Y. \& LEVY, A. - Foundations of set theory, Studies in Logic and the Foundations ofMathematics, vol. 67,NorthHolland, Amsterdam,New York, Oxford, 1958, 1973.

[14] GLIVENKO, V. - « Sur quelques points de la logique de M. Brouwer », in Bulletins de la classe des sciences, 5, vol. 15, Academie Royale de Belgique, 1929, p. 183-188.

[15] LEHRER, K. - « Why not Skepticism ? », The Philosophical Forum 2 (1971), no. 3, p. 283-298, Reprinted in Essays on Knowledge and Justification, George S. Pappas and Marshall Swain (eds), (Cornell, 1978), pp. 346-363.

[16] LÖB, M.H. - «Embedding First Order Predicate Logic in Fragments of Intuitionistic Logic », The Journal of Symbolic Logic 41 (1976), no. 4, p. 705-718.

[17] MCKINSEY, J.C.C \& TARSKI, A. - « Some theorems about the sentential calculi of Lewis and Heyting », The Journal of Symbolic Logic 13 (1948), p. 1-15.

[18] MOTOHASHI, N. - " A faithful interpretation of intuitionistic predicate logic in classical predicate logic », CommentariiMathematici Universitatis Sancti Pauli 21 (1972), p. 11-23.

[19] NEGRI, S. \& VON PLATO, J. - Structural Proof Theory, Cambridge University Press, Cambridge, 2001.

[20] QUINE, W.V.O. - «On What There Is », [21, pp. 1-19], tr. fr. pp. 25-48.

[21], From a Logical Point of View, Cambridge University Press, Cambridge Mass.,

London, England, 1953, 1961, 1980, tr. fr. Laugier (ed.), Du point de vue logique, Vrin, Paris, 2003.

[22] , Word and Object, The M.I.T. Press, Cambridge Mass., 1960, tr. fr. Gochet, Le Mot

et la Chose, Flammarion, Paris, 1960.

[23], Set theory and its logic, The Belnap Press ofHarvardUniversity Press, Cambridge, Mass., 1963, 1969.

[24] , « Russell's Ontological Development », The Journal of Philosophy 63 (1966), no. 21, p. 657667.

[25], Ontological Relativity and Other Essays, Columbia University Press, New York, London, 1969, tr. fr. Largeault, Relativité de l'ontologie et autres essais, Aubier-Montaigne, Paris, 1977.

[26] , Philosophy of Logic, Prentice Hall, Englewood Cliffs, 1970, tr. fr. Largeault, Aubier-Montaigne, Paris, 1975. 
AL-MUKHATABAT ISSN 1737-6432 Numéro - issue 11 /July - Juillet 2014

[27], Pursuit of Truth, Harvard University Press, New-York, 1990, La poursuite de la vérité, trad. fr. Clavelin, Seuil, Paris, 1993.

[28] R. DAVID \& K. NOUR \& C. RAFFALLI - Introduction à la logique (théorie de la démonstration, cours et exercices corrigés), Dunod, Paris, 2001, 2003.

[29] RASHED, R. \& PELLEGRIN, P. (éd.) - Philosophie des mathématiques et théorie de la connaissance - L'oeuvre de Jules Vuillemin, Collection Sciences dans l'Histoire, Paris, Albert Blanchard, 2005.

[30] RUSSELL, B. - The Principles of Mathematics, Allen and Unwin, London, 1903, 1st ed. Cambridge University Press ; tr. fr. Roy, Ecrits de logique philosophique, PUF, Paris, 1989.

[31] SØRENSEN, M.H. \& URZYCZYN, P. - « A Syntactic Embedding of Predicate Logic into Second-Order Propositional Logic », Notre Dame Journal of Formal Logic 51 (2010), no. 457-473.

[32] VIDAL-ROSSET, J. - « L'intuitionnisme de Gaston Bachelard », in Actualité et Postérités de Gaston Bachelard (Paris) (Nouvel, P., éd.), Presses Universitaires de France, 1997, p. 117-138.

[33], «Philosophy of Mathematics and Ontological Commitments », Journal of Philosophy of Science Society 33 (2000), no. 1, p. 69-81, Tokyo, Japon.

[34], « Some Remarks on Relations between Proofs and Games », in Festschrift for

Gerhard Heinzmann (Bour \& Rebuschi \& Rollet, éd.), vol. Tribute Series Editor, 2010, coauthored with Galmiche D. and Larchey-Wendling, D., p. 335-353.

[35], « Stable Philosophical Systems and Radical Anti-Realism », in The Realism-Antirealism Debate in the Age of Alternative Logics (Marion, M. \& Rahman, S. \& Primiero, G., éd.), vol. 23, Logic, Epistemology \& Unity of Science, no. 1, Springer, 2011, p. 313-324.

[36], "L'argument de Russell-Tennant », in Autour des PrincipiaMathematica, B. Russell et A. N. Whitehead 1910-1913 (Dijon) (A. Guay, éd.), Éditions universitaires de Dijon, 2011, p. 149-177.

[37] VON PLATO, J. - « Proof Theory of Full Classical Propositional Logic », ms., 1998.

[38] VUILLEMIN, J. - « Etre et Choix - Eléments de philosophie réaliste », Manuscrit inédit des Archives Vuillemin, Université de Lorraine, Archives Poincaré, UMR 7117 du CNRS, Nancy.

[39], «Les formes fondamentales de la classification : un essai de classification », in Recherches sur la philosophie et le langage - Langage et philosophie des sciences (Grenoble), vol. 4, Cahier du groupe de recherches sur la philosophie et le langage - Département de philosophie - Université de Grenoble, Services des Publications de l'Université des Sciences

Sociales de Grenoble, 1984, p. 9-30.

[40], Nécessité ou Contingence, l'aporie de Diodore et les systèmes philosophiques, Minuit, Paris, 1984.

[41], What are philosophical systems ?, Cambridge University Press, Cambridge, 1986.

[42], Necessity or Contingency - The Master Argument, no. Lecture Notes 56, CSLI Publications, Stanford, 1996.

[43], « Formalisme et réflexion philosophique », Bulletin de la société française de philosophie 94e année (2000), no. 3, Séance du 25 mars 2000.

[44] WALTON, D.N. - «Burden of Proof », Argumentation 2 (1988), no. 2, p. 233-254. 